\title{
Comparison of the Effect of Phenylalanine Arginine Beta Naphthylamide (PAßN) and Curcumin on Minimum Inhibitory Concentration of Aminoglycosides on Pseudomonas aeruginosa Clinical Isolates
}

\author{
Parisa Charkhi $^{1,2}$ (D), Mohammad Reza Haghshenas ${ }^{1,2}$ (D), Bahman Mirzaei ${ }^{3}(\mathbb{D})$

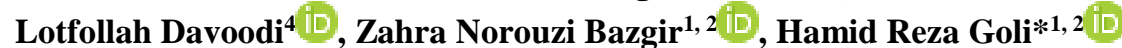

1. Molecular and Cell Biology Research Centre, Faculty of Medicine, Mazandaran University of Medical Sciences, Sari, Iran

2. Dept. of Medical Microbiology and Virology, Faculty of Medicine, Mazandaran University of Medical Sciences, Sari, Iran

3. Dept. of Medical Microbiology and Virology, School of Medicine, Zanjan University of Medical Sciences, Zanjan, Iran

4. Antimicrobial Resistance Research Center, Faculty of Medicine, Mazandaran University of Medical Sciences, Sari, Iran

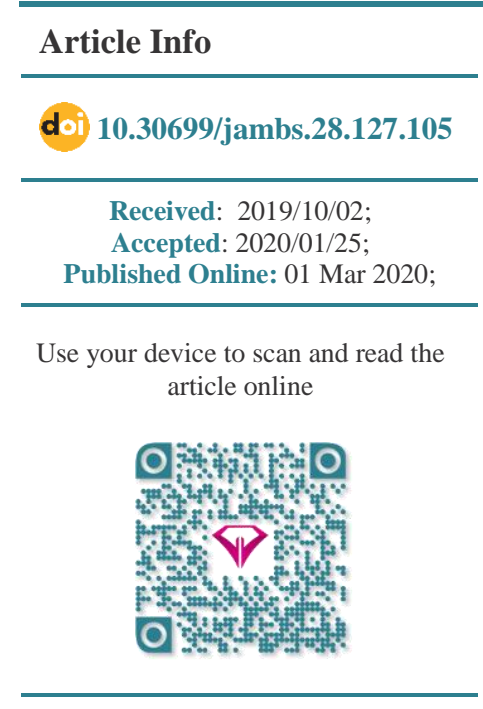

Corresponding Information: Hamid Reza Goli,

Molecular and Cell Biology Research Centre, Faculty of Medicine, Mazandaran University of Medical Sciences, Sari, Iran

E-Mail: goli59@gmail.com

\begin{abstract}
Background \& Objective: Efflux pump inhibitors (EPIs) can block efflux pumps and are helpful in potentiating the activity of aminoglycosides against Pseudomonas aeruginosa. The present study compared the effects of phenylalanine-arginine beta naphthylamide $(\mathrm{PA} \beta \mathrm{N})$ and curcumin on aminoglycoside minimum inhibitory concentration (MIC) on Pseudomonas aeruginosa clinical isolates.
\end{abstract}

Materials \& Methods: For this descriptive-analytical study, 100 clinical isolates of Pseudomonas aeruginosa were collected and identified by differential diagnostic tests. The MICs of amikacin, gentamicin, and tobramycin were evaluated before and after adding EPIs using a micro-broth dilution test.

Results: The bacteria were isolated from different types of samples, including urine (26 isolates), sputum (37 isolates), ulcers (20 isolates), catheters (eight isolates), blood (five isolates), feces (two isolates), and eyes (two isolates). Overall, $60 \%$ of the isolates were obtained from males (mean age $=47.85)$, and $40 \%$ from females (mean age $=$ 44.76). In the MIC test, 11 (25.5\%), 15 (34.8\%), and 18 (41.8\%) isolates were resistant to amikacin, gentamicin, and tobramycin, respectively. Significant reductions in the MICs of amikacin, gentamicin, and tobramycin were observed after adding curcumin in 54-100\% of aminoglycoside-resistant isolates, while fewer changes in the MICs of aminoglycosides were seen against these clinical isolates after adding PA $\beta \mathrm{N}(36-55 \%)$.

Conclusion: Curcumin and PA $\beta \mathrm{N}$ can potentiate the effect of aminoglycosides on clinical isolates of Pseudomonas aeruginosa and change their susceptibility pattern due to efflux pump inhibition. However, our outcomes detected that curcumin was more effective than the PA $\beta \mathrm{N}$ against the aminoglycoside-resistant isolates of $P$. aeruginosa.

Keywords: Curcumin, Efflux pump, Inhibitor, Phenylalanine arginine beta naphthylamide, Pseudomonas aeruginosa

\begin{tabular}{|c|c|}
\hline (c) (1) (8) & $\begin{array}{l}\text { Copyright } \odot 2020 \text {, This is an original open-access article distributed under the terms of the Creative Commons Attribution-noncommercial } 4.0 \text { International License which permits } \\
\text { copy and redistribution of the material just in noncommercial usages with proper citation. }\end{array}$ \\
\hline
\end{tabular}

\section{Introduction}

Pseudomonas aeruginosa is an opportunistic Gramnegative rod that causes nosocomial infections such as leukemia and cystic fibrosis (CF) in susceptible individuals (1) and accounts for nearly $30 \%$ of antibiotic-resistant nosocomial infections $(1,2)$. The ability to survive under adverse environmental conditions (including drought)—along with the acquired and intrinsic factors of antibiotic resistance, such as receiving antibiotic resistance genes, altering membrane permeability, enhancing expression of efflux pumps, achieving secondary metabolic pathways, the production of enzymes that destroy and deactivate antibiotics, the acquisition of integrons carrying resistance genes, etc.-make this bacterium a lethal pathogen $(3,4)$. Efflux pumps are one of the most significant mechanisms of resistance in $P$. aeruginosa. They are capable of excreting toxic substances such as antibiotics, drugs, and chemicals, and they can secrete cellular products from the bacterial cell $(4,5)$. These systems prevent the formation of appropriate concentrations of toxic substances that inhibit bacteria and act as a defense mechanism against environmentally harmful substances, thus promoting bacterial survival in different environments $(5,6)$. Based on the similarity and sequence of amino acids, efflux systems are divided into five large families from 
which the clinically important resistance nodulation cell division (RND) family (which contains multidrug resistance efflux pumps) utilizes ATP energy to move the substrates $(5,7)$. These efflux systems can extrude a wide range of antibiotics, including fluoroquinolones, aminoglycosides, tetracyclines, and chloramphenicol (7). Aminoglycosides play important roles in the treatment of infections caused by $P$. aeruginosa, especially in CF patients $(8,9)$. A combination of aminoglycosides and beta-lactam is used to treat the severe infections caused by this bacterium, but unfortunately, resistance to these antibiotics is increasing (10). Studies have shown that by blocking efflux pumps in resistant strains of bacteria, the efficacy of antibiotics and biocides is significantly increased (11), while various materials have been identified as inhibitors of the performance of efflux pumps (12). Phenylalanine-arginine betanaphthylamide $(\mathrm{PA} \beta \mathrm{N})$ is one of the most significant identified inhibitors of RND efflux pumps in Gramnegative bacteria $(11,12)$. The mechanism of action of this substance is competitive inhibition, as efflux pumps in the bacteria detect and remove it instead of the target antibiotics (fluoroquinolones, mainly ciprofloxacin and levofloxacin) from the cells. As a result, antibiotics remain in the cell and increase in concentration (13). Curcumin is also a natural inhibitor derived from turmeric and has been proven to have anti-cancer, anti-inflammatory, and antimicrobial effects against viruses, bacteria, and pathogenic fungi (14). It also has a blocking effect on efflux pumps (15). The mechanism of its effect on efflux pumps is not yet fully understood, but in bacteria such as Escherichia coli and Bacillus subtilis, it inhibits cell division by preventing FtsZ polymerization and its assembly. It is also known to block Helicobacter pylori by inhibiting enzymes called matrix metalloproteinase 3 and matrix metalloproteinase 9, thereby preventing the inflammatory responses induced by this bacterium (14). Curcumin is not very toxic, and its pharmacological effects are well known (15).

Studies on non-toxic inhibitors and their effects on the minimum inhibitory concentration (MIC) of aminoglycoside against $P$. aeruginosa are very important in the treatment of infections caused by multidrug-resistant strains of this bacterium. Therefore, in this study, we compared the MICs of $\mathrm{PA} \beta \mathrm{N}$ and curcumin on clinical isolates of $P$. aeruginosa and $\mathrm{t}$ investigated their synergistic effects in combination with aminoglycosides.

\section{Materials and Methods}

\section{Cultivation and Isolation of Bacteria}

In this descriptive-analytical study, 100 clinical isolates of $P$. aeruginosa were collected from patients hospitalized from September 2018 to September 2019 in different teaching and therapeutic hospitals of Mazandaran province, which is in northern Iran. The studied bacteria were isolated from sputum (37 isolates), urine (26 isolates), ulcers (19 isolates), intravenous catheters (eight isolates), blood (five isolates), feces (two isolates), ocular secretions (two isolates), and diabetic foot ulcer secretions (one isolate). The 100 clinical samples were collected from patients hospitalized in intensive care units (ICUs) $(n=48)$, emergency rooms $(n=13)$, burn wards $(n=9)$, cardiac care units (CCUs) $(n=6)$, pediatric units $(n=6)$, internal medicine units $(n=4)$, operating rooms $(n=3)$, men's sections $(n=3)$, surgical sections $(n=3)$, women's sections $(n=2)$, neurology departments $(n=2)$, and oncology departments $(\mathrm{n}=1)$. All clinical isolates of $P$. aeruginosa were identified on the basis of morphology and colony odor, Gram staining, pigment production, biochemical test results, positive oxidase, and catalase tests, $\mathrm{O} / \mathrm{F}$ test results, and the ability to grow at $42^{\circ} \mathrm{C}$ (16). Finally, pure isolates were cultured in a Trypticase soy broth (TSB) medium (Merck, Germany) containing $10 \%$ glycerol and were transferred to $\mathrm{a}-20^{\circ} \mathrm{C}$ freezer for subsequent work.

\section{Determination of Minimum Inhibitory Concentration (MIC) of Aminoglycosides by the Micro-Broth Dilution Method}

The MIC of amikacin, gentamicin, and tobramycin (Sigma, Germany) against the clinical isolates of $P$. aeruginosa was determined based on the guidelines of the Clinical and Laboratory Standards Institute (CLSI) (17) using a micro-broth dilution method. Then, the MIC tests of these aminoglycosides were repeated after adding $50 \mu \mathrm{g} / \mathrm{mL}$ of each efflux pump inhibitor (i.e., PA $\beta N$ (Sigma, Germany) and curcumin (Sigma, Germany)) to the Müller-Hinton broth medium (Merck, Germany). After adding these inhibitors either separately or in combination with the Müller-Hinton broth medium (which contained definite concentrations of the antibiotics), the MICs were measured and recorded. The difference between the two MICs (i.e., with and without the inhibitors) was considered an indicator of the function of the efflux pump inhibitors. The reduction of the MIC by adding the inhibitors indicates their effect on efflux pumps and vice versa.

\section{Results}

\section{Clinical Information}

In this study, 100 clinical isolates of Pseudomonas aeruginosa were collected from patients hospitalized in Imam Khomeini (a general hospital) (40 isolates), Zare (a burn center) (11 isolates), BuAli Sina (a pediatric hospital) (17 isolates), Fatemeh al-Zahra (a heart diseases center) (10 isolates), and Razi (an infectious hospital) ( 22 isolates). The bacteria were isolated from different types of samples, including urine (26 isolates), sputum (37 isolates), ulcers (20 isolates), catheters (eight isolates), blood (five isolates), feces 
Table 1. MIC values of aminoglycosides in the absence of efflux pump inhibitors

\begin{tabular}{ccccccccccccc} 
& \multicolumn{10}{c}{ Number of isolates with different MIC ranges $(\mu \mathrm{g} / \mathrm{mL})$} \\
\hline MIC ranges & 1024 & 512 & 256 & 128 & 64 & 32 & 16 & 8 & 4 & 2 & $<0.5$ \\
Gentamicin & 4 & 2 & 3 & 2 & 3 & 1 & - & - & - & - \\
\hline Tobramycin & 2 & 3 & 3 & 3 & 3 & 3 & 1 & - & - & - & - \\
Amikacin & 4 & 2 & - & 2 & 3 & - & - & - & - & - & - \\
\hline
\end{tabular}

Table 2. MIC values of aminoglycosides in the presence of efflux pump inhibitors

\begin{tabular}{ccccccc}
\hline \multicolumn{5}{c}{ No. $(\%)$ of isolates with fold change of aminoglycosides' MIC with } \\
\hline $\begin{array}{c}\text { MIC Fold } \\
\text { change }\end{array}$ & Gentamicin & Tobramycin & Amikacin & Gentamicin & Tobramycin & Amikacin \\
\hline $\begin{array}{c}\text { Unchanged } \\
\text { one-fold } \\
\text { reduction }\end{array}$ & $9(60 \%)$ & $8(44.4 \%)$ & $7(63.6 \%)$ & - & - & $5(45.4 \%)$ \\
\hline $\begin{array}{c}\text { two-fold } \\
\text { reduction }\end{array}$ & $1(26.6 \%)$ & $8(44.4 \%)$ & $3(27.2 \%)$ & $1(6.6 \%)$ & $7(38.8 \%)$ & $2(18.1 \%)$ \\
$\begin{array}{c}\text { three-fold } \\
\text { reduction }\end{array}$ & - & - & - & $5(33.3 \%)$ & $5(27.7 \%)$ & $1(9.09 \%)$ \\
\hline $\begin{array}{c}>\text { three-fold } \\
\text { reduction }\end{array}$ & $1(6.6 \%)$ & $1(5.5 \%)$ & $1(9.09 \%)$ & $4(26.6 \%)$ & $3(16.6 \%)$ & $3(27.2 \%)$ \\
\hline $\begin{array}{c}\text { Total resistant } \\
\text { isolates }\end{array}$ & 15 & 18 & 11 & $15 \%)$ & 18 & 11 \\
\hline
\end{tabular}

(two isolates), and eyes (two isolates). Overall, $60 \%$ of the isolates were obtained from males (mean age $=$ 47.85 years), and $40 \%$ were obtained from females (mean age $=44.76$ years).

\section{The Minimum Inhibitory Concentration of Aminoglycosides}

In the MIC test, $11(25.5 \%), 15(34.8 \%)$, and 18 $(41.8 \%)$ isolates were resistant to amikacin, gentamicin, and tobramycin, respectively (Table 1). The MIC results after adding efflux pump inhibitors (PA $\beta \mathrm{N}$ and curcumin) are shown in Table 2.

The results are as follows:

All 18 tobramycin-resistant isolates showed MIC reductions with curcumin, while seven isolates $(38.8 \%)$ showed a one-fold reduction in MIC, five isolates $(27.7 \%)$ exhibited a two-fold reduction, three isolates (16.6\%) showed a three-fold reduction, and another three isolates (16.6\%) showed more than a three-fold MIC reduction. Among the 18 isolates that were resistant to tobramycin, MIC decreased with phenylalanine arginine beta naphthylamide in 10 isolates $(55.5 \%)$. Only one isolate $(10 \%)$ exhibited more than a three-fold MIC reduction with this EPI. $\mathrm{PA} \beta \mathrm{N}$ was completely ineffective on eight isolates and did not change the MIC of tobramycin in these isolates.

A similar situation was observed for gentamicin, in that out of 15 resistant isolates, all showed MIC reductions with curcumin, while five $(33.3 \%)$ isolates showed a three-fold reduction and four $(62.6 \%)$ showed agreater-than-three-fold reduction in gentamicin MIC in combination with curcumin. However, only six (40\%) gentamicin-resistant isolates exhibited an MIC reduction after phenylalanine arginine beta naphthylamide was added, and this EPI was ineffective in gentamicin MIC against nine (60\%) resistant isolates.

The effect of curcumin on amikacin MIC depletion was less than that of the other two aminoglycosides, which were effective on six $(54.5 \%)$ resistant isolates, while three isolates $(50 \%)$ exhibited more than a threefold reduction in amikacin MIC with curcumin. However, the inhibitory potency of PA $\beta \mathrm{N}$ in combination with amikacin was relatively weak, and four isolates $(36.3 \%)$ that were resistant to amikacin, thus causing a reduction in antibiotic MIC, showed a one-fold decrease in MIC.

Out of 15 gentamicin-resistant isolates, four (26.6\%) isolates changed as the sensitive isolates after adding curcumin, while one isolate $(6.6 \%)$ was within the range of intermediate-resistant in combination with this EPI. Also, after the addition of curcumin to the MIC tests of 11 amikacin-resistant isolates, one isolate $(9.09 \%)$ was changed to become susceptible to this antibiotic, and two others (18.18\%) were reformed into intermediate resistant isolates. Moreover, after adding curcumin to 18 tobramycin resistant isolates, two isolates $(11.11 \%)$ were transformed into susceptible 
isolates, and another two (11.11\%) changed into intermediate resistant isolates.

Also, after the addition of PA $\beta \mathrm{N}$ to the MIC tests of tobramycin resistant isolates, just one isolate $(5.5 \%)$ was found to be susceptible, and one other $(5.5 \%)$ changed into an intermediate resistant isolate. However, PA $\beta \mathrm{N}$ had no significant effect on the gentamicin- and amikacin-resistant isolates in the present study.

\section{Discussion}

Pseudomonas aeruginosa is an important pathogen in nosocomial infections, especially in ICU and burn ward patients (18). This organism increases resistance to aminoglycosides by employing various mechanisms such as the production of drug-modifying enzymes, increased expression of efflux pumps, and uptake of aminoglycoside resistance genes (4). In the present study, the number of isolates resistant to aminoglycosides (gentamicin, tobramycin, and amikacin) was estimated at $41 \%$, which is consistent with some other studies in Iran $(19,20)$. Amikacin resistance was significantly lower than gentamicin and tobramycin in this study. One possible reason for the low rate of amikacin resistance is the widespread use of gentamicin and tobramycin in this region (21). Efflux pumps are important for the development of aminoglycoside resistance in $P$. aeruginosa, and it is necessary to apply a suitable strategy to combat them. One such strategy is the use of efflux pump inhibitors with antibiotics (22). The MIC results of the tested antibiotics showed that after the addition of efflux pump inhibitors (curcumin and $\mathrm{PA} \beta \mathrm{N}$ ), the efflux pump inhibited $36-100 \%$ of the aminoglycosideresistant isolates, and the MIC of aminoglycosides decreased in these isolates (Table 2). We found that curcumin reduced the MIC in $100 \%$ of gentamicin- and tobramycin-resistant isolates. The MIC results after adding phenylalanine-arginine beta naphthylamide also showed that it had a beneficial effect on the reduction of tobramycin MIC, although this was only observed in $55 \%$ of the resistant isolates.

To date, several studies have examined efflux pump inhibitors and their effect on increasing the MIC of different antibiotics. Many of these studies have emphasized the increased antimicrobial activity of various antibiotics in addition to efflux pump inhibitors (11,23-25). Sonnet et al. showed that PA $\beta N$ can reduce the MIC of ciprofloxacin in $P$. aeruginosa (26). However, most of the aminoglycoside-resistant isolates in our study did not show a significant change in the MIC values of gentamicin, tobramycin, or amikacin after adding a PA $\beta N$ inhibitor. Our results showed that $\mathrm{PA} \beta \mathrm{N}$ did not significantly enhance the antimicrobial activity of the tested antibiotics. Another study in Turkey on Acinetobacter baumannii isolates confirmed our results and showed that despite the inhibitory effect of PA $\beta \mathrm{N}$ on enhancing the activity of some antibiotics, this inhibitor does not have a significant effect on aminoglycosides' action against the clinical isolates of $P$. aeruginosa (27).

Increasing drug resistance has led researchers to try to find alternative or complementary herbal medicines to inhibit the growth of these pathogens, and many studies have been conducted in this area. A study by Sisay et al. (2019) showed that the extract of Myrtus significantly reduces the growth inhibitory zone diameter of $P$. aeruginosa in laboratory (28). The results of another study carried out by Chakotiya et al. (2016) on Glycyrrhiza glabra exhibited the effective role of this plant's extract on inhibiting the growth of $P$. aeruginosa clinical isolates (29). Additionally, Morita et al. found that barberry extract specifically inhibits aminoglycoside-resistant $P$. aeruginosa strains (30). Similar to other researchers' findings, we observed a significant antibacterial effect of curcumin against clinical isolates of $P$. aeruginos $a$ in the present study.

Ballard et al. showed that the use of natural curcumin in combination with antibiotics can decrease the MICs of antibiotics (31). Another study showed that as the concentration of curcumin rises, its effectiveness as an inhibitor increases (13). In our study, 54 to $100 \%$ of aminoglycoside-resistant isolates showed a decrease in the MICs of these antibiotics after curcumin addition. This suggests that this substance has a greater effect than PA $\beta \mathrm{N}$ as an inhibitor, which has been confirmed by other studies $(13,15)$.

On the other hand, Eshra et al. found a significant difference in the reduction of the gentamicin MIC after adding PA $\beta \mathrm{N}$ and curcumin (13). Similar to the abovementioned study, $100 \%$ of gentamicin-resistant isolates showed a significant decline in MIC after adding curcumin in the present study, while PA $\beta \mathrm{N}$ had no significant effect. One possible reason for the observed differences in the effect of inhibitors on the efficiency of different antibiotics is the presence of highly diverse drug resistance mechanisms in Pseudomonas aeruginosa, of which efflux pumps are only one.

\section{Conclusion}

A phenotypic study on the expression of efflux systems using its inhibitors and reductions of the MIC of Pseudomonas aeruginosa clinical isolates in the present study showed that as a chemical inhibitor, natural curcumin had a better inhibitory effect on aminoglycoside-resistant clinical isolates than PA $\beta N$. However, other studies have reported that PA $\beta \mathrm{N}$ has a strong effect on decreasing the MICs of other antibiotics. In addition, the inhibitory effect of curcumin with gentamicin was better than that of tobramycin and amikacin, and it seems that gentamicin combined with curcumin (as an adjuvant) can be used to treat Pseudomonas aeruginosa infections and reduce the gentamicin resistance rate. However, further in vivo investigations are required to confirm this result. Since 
curcumin is a natural substance, it can be substituted for chemicals that inhibit efflux pumps.

\section{Acknowledgments}

We thank the laboratory staffs of BuAli Sina, Imam Khomeini, Zare, Fatemeh Zahra and Razi hospitals for providing patients' information and the collection of the clinical isolates.

\section{Conflict of Interest}

Authors declared no conflict of interest.

\section{Ethical Approval}

This research was approved by ethics committee of Mazandaran University of Medical Sciences, Sari, Iran, with ethical code IR.MAZUMS.REC.1397.1431.

\section{References}

1. Jones RN, Stilwell MG, Rhomberg PR, Sader HS. Antipseudomonal activity of piperacillin/tazobactam: more than a decade of experience from the SENTRY Antimicrobial Surveillance Program (1997-2007). DiagnMicrobiol Infect Dis. 2009;65(3):331-4. [DOI:10.1016/j.diagmicrobio.2009.06.022]

2. Gailienè G, Pavilonis A, Kareivienè V. The peculiarities of Pseudomonas aeruginosa resistance to antibiotics and prevalence of serogroups. Medicina. 2007;43(1):36. [DOI:10.3390/medicina43010005]

3. Kashfi M, Hashemi A, Eslami G, Amin MS, Tarashi $\mathrm{S}$, Taki E. The prevalence of aminoglycosidemodifying enzyme genes among Pseudomonas aeruginosa strains isolated from burn patients. Arch Clin Infect Dis. 2017;12(1): e40896; 10.5812/archcid.40896.

[DOI:10.5812/archcid.40896]

4. Hassett D, Charniga L, Bean K, Ohman D, Cohen M. Response of Pseudomonas aeruginosa to pyocyanin: mechanisms of resistance, antioxidant defenses, and demonstration of a manganese-cofactored superoxide dismutase. Infect Immun. 1992;60(2):328-36. [DOI:10.1128/IAI.60.2.328-336.1992]

5. Poole K. Efflux-mediated antimicrobial resistance. J AntimicrobChemother.

2005;56(1):20-51. [DOI:10.1093/jac/dki171]

6. Poole K. Mechanisms of bacterial biocide and antibiotic resistance. J App Microbiol. 2002;92:55S64S. [DOI:10.1046/j.1365-2672.92.5s1.8.x]

7. Poole K. Multidrug efflux pumps and antimicrobial resistance in Pseudomonas aeruginosa and related organisms. J MolMicrobiol Biotech. 2001;3(2):25564.

8. Forge A, Schacht J. Aminoglycoside antibiotics. Audiology and Neurotology. 2000;5(1):3-22. [DOI:10.1159/000013861]
9. Poole K. Aminoglycoside resistance in Pseudomonas aeruginosa. Antimicrob Agents Chemother. 2005;49(2):479-87. [DOI:10.1128/AAC.49.2.479487.2005]

10. Driscoll JA, Brody SL, Kollef MH. The epidemiology, pathogenesis and treatment of Pseudomonas aeruginosa infections. Drugs. 2007;67(3):351-68. [DOI:10.2165/00003495-200767030-00003]

11. Pannek S, Higgins PG, Steinke P, Jonas D, Akova M, Bohnert JA, et al. Multidrug efflux inhibition in Acinetobacterbaumannii: comparison between 1-(1naphthylmethyl)-piperazine and phenyl-arginine- $\beta$ naphthylamide. J Antimicrob Chemother. 2006;57(5):970-4. [DOI:10.1093/jac/dkl081]

12. Lomovskaya O, Bostian KA. Practical applications and feasibility of efflux pump inhibitors in the clinic-a vision for applied use. Bioch Pharm. 2006;71(7):9108. [DOI:10.1016/j.bcp.2005.12.008]

13. Eshra KA, Shalaby MM. Efflux pump inhibition effect of curcumin and phenylalanine arginyl $\beta$ naphthylamide $(\mathrm{PA} \beta \mathrm{N})$ against multidrug resistant Pseudomonas aeruginosa isolated from burn infections in Tanta University Hospitals. Egypt J Med Microbiol. 2017;38(5782):1-7 [DOI:10.12816/0046279]

14. ZorofchianMoghadamtousi S, Abdul Kadir H, Hassandarvish P, Tajik H, Abubakar S, Zandi K. A review on antibacterial, antiviral, and antifungal activity of curcumin. BioMed Res Int. 2014;2014: 112; [DOI:10.1155/2014/186864.]

15. Negi N, Prakash P, Gupta ML, Mohapatra TM. Possible role of curcumin as an efflux pump inhibitor in multi drug resistant clinical isolates of Pseudomonas aeruginosa. J ClinDiagn Res: JCDR. 2014;8(10):

4-8. [DOI:10.7860/JCDR/2014/8329.4965]

16. Greaves P. Bailey \& Scott's Diagnostic Microbiology. J Med Microbiol. 1991;35(2):125-6. [DOI:10.1099/00222615-35-2-125b]

17. CLSI. Clinical and Laboratory Standards Institute: Performance standards for antimicrobial susceptibility testing: Twenty-eighth informational supplement, M100-S28. Wayne, PA: Clinical and Laboratory Standards Institute (CLSI) 2018; vol. 35.

18. Beig M, Arabestani MR. Investigation of MexABOprM efflux pump gene expression in clinical isolates of Pseudomonas aeruginosa isolated from Intensive Care Unit. Iran J Med Microbiol. 2019;13(2):142-50. [DOI:10.30699/ijmm.13.2.142]

19. Zarei O, Shokoohizadeh L, Hossainpour H, Alikhani MY. Molecular analysis of Pseudomonas aeruginosa isolated from clinical, environmental and cockroach sources by ERIC-PCR. BMC Res Notes. 2018;11(1):1-7. [DOI:10.1186/s13104-018-3765-z] 
20. Sharifi H, Pouladfar G, Shakibaie MR, Pourabbas B, Mardaneh J, Mansouri S. Prevalence of $\beta$-lactamase genes, class 1 integrons, major virulence factors and clonal relationships of multidrug-resistant Pseudomonas aeruginosa isolated from hospitalized patients in southeast of Iran. Iran J Basic Med Sci. 2019;22(7):806-12.

21. Goli HR, Nahaei MR, Rezaee MA, et al. Prevalence and molecular characterization of Class 1 integrons among clinical isolates of Pseudomonas aeruginosa in Northwest of Iran. Mol Genet MicrobiolVirol. 2017;32(2):109-15.

\section{[DOI:10.3103/S0891416817020057]}

22. AfsharYavari S, Rota S, Caglar K, Fidan I. Efflux pump inhibitors effect on the minimum inhibitory concentration of Benzalkonium Chloride and Chlorhexidine in Acinetobacter baumannii strains isolated from hospitalized inpatients in Gazi hospital, Ankara. J UrmiaUniv Med Sci. 2016;27(5):393-401.

23. Peleg AY, Adams J, Paterson DL. Tigecycline efflux as a mechanism for nonsusceptibility in Acinetobacterbaumannii. Antimicrob Agents Chemother. 2007;51(6):2065-9.

[DOI:10.1128/AAC.01198-06]

24. Vecchione JJ, Alexander B, Sello JK. Two distinct major facilitator superfamily drug efflux pumps mediate chloramphenicol resistance in Streptomyces coelicolor. Antimicrob Agents Chemother. 2009;53(11):4673-7. [DOI:10.1128/AAC.00853-09]

25. Li L, Li Z, Guo N, et al. Synergistic activity of 1-(1naphthylmethyl)-piperazine with ciprofloxacin against clinically resistant Staphylococcus aureus, as determined by different methods. Lett App Microbiol. 2011;52(4):372-8.

[DOI:10.1111/j.1472765X.2011.03010.X]

26. Sonnet P, Izard D, Mullié C. Prevalence of effluxmediated ciprofloxacin and levofloxacin resistance in recent clinical isolates of Pseudomonas aeruginosa and its reversal by the efflux pump inhibitors 1-(1naphthylmethyl)-piperazine and phenylalanine- arginine- $\beta$-naphthylamide. IntJAntimicrob Agents. 2012;39(1):77-80.

[DOI:10.1016/j.ijantimicag.2011.08.005]

27. Dal T, Aksu B, Pagès J-M, Over-Hasdemir U. Expression of the adeB gene and responsiveness to 1(1-naphthylmethyl)-piperazine and phenylalanylarginyl- $\beta$-naphthylamide in clinical isolates of Acinetobacter baumannii. J AntimicrobChemother. 2013;68(5):1200-2. [DOI:10.1093/jac/dks511]

28. Sisay M, Bussa N, Gashaw T, Mengistu G. Investigating In Vitro Antibacterial Activities of Medicinal Plants Having Folkloric Repute in Ethiopian Traditional Medicine. J Evidence-based Integ Med. 2019;24:2515690X19886276. [DOI:10.1177/2515690X19886276]

29. Chakotiya AS, Tanwar A, Narula A, Sharma RK. Alternative to antibiotics against Pseudomonas aeruginosa: Effects of Glycyrrhiza glabra on membrane permeability and inhibition of efflux activity and biofilm formation in Pseudomonas aeruginosa and its in vitro time-kill activity. Microb Path. 2016;98:98-105.

[DOI:10.1016/j.micpath.2016.07.001]

30. Morita Y, Nakashima K-i, Nishino K, Kotani K, Tomida $\mathrm{J}$, Inoue $\mathrm{M}$, et al. Berberine is a novel type efflux inhibitor which attenuates the MexXYmediated aminoglycoside resistance in Pseudomonas aeruginosa. Front Microbiol. 2016;7:1223. [DOI:10.3389/fmicb.2016.01223]

31. Ballard E, Coote PJ. Enhancement of antibiotic efficacy against multi-drug resistant Pseudomonas aeruginosa infections via combination with curcumin and 1-(1-Naphthylmethyl)-piperazine. J Antimicrob Agents. 2016;2: 1000116. [DOI:10.4172/24721212.1000116.]

\section{How to Cite This Article:}

Charkhi P, Haghshenas M R, Mirzaei B, Davoodi L, Norouzi Bazgir Z, Goli H R. Comparison of the Effect of Phenylalanine Arginine Beta Naphthylamide $(\mathrm{PA} \beta \mathrm{N})$ and Curcumin on Minimum Inhibitory Concentration of Aminoglycosides on Pseudomonas aeruginosa Clinical Isolates. J Adv Med Biomed Res. 2020; 28 (127) :105-110

\section{Download citation:}

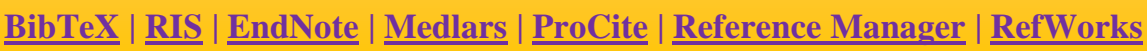

Send citation to:

Mendeley 2 Zotero (i) RefWorks $\underline{\text { RefWorks }}$ 